\title{
Estado nutricional, hábitos de alimentación y de estilo de vida en vegetarianos de Asunción y Gran Asunción, Paraguay
}

\author{
Nutritional status, eating habits and lifestyle factors among vegetarians \\ from Asunción and Great Asunción, Paraguay
}

\section{RESUMEN}

Una dieta vegetariana se define por la exclusión total o parcial de alimentos de origen animal. Cuando es planificada adecuadamente puede prevenir y tratar enfermedades. Sin embargo, una dieta restrictiva en ciertos alimentos debe ser evaluada. El propósito de esta investigación fue describir parámetros antropométricos, hábitos de alimentación y de estilo de vida en ovo-lácteo-vegetarianos y veganos y explorar diferencias en la ingesta de nutrientes críticos entre ambos grupos. Estudio descriptivo de corte transversal, realizado en ovo-lácteo-vegetarianos y veganos de Asunción, Paraguay, durante el 2015. Se evaluaron parámetros antropométricos,, ingesta y hábitos de estilo de vida saludable. Participaron 17 ovo-lácteo-vegetarianos y 14 veganos. El 67,7\% $(n=21)$ se encontró normopeso, el $29 \%(n=9)$ presentó exceso de peso, y el 3,2\% ( $n=1)$ bajo peso. Se presentó una ingesta inferior a los requerimientos en energía, carbohidratos, proteínas y grasas en ambos grupos de vegetarianos, e ingesta muy limitada de calcio y vitamina B12 en veganos. Las mujeres presentaron ingesta muy por debajo de las recomendaciones para hierro y calcio. Ninguna mujer vegana cumplió con la recomendación dietaria de vitamina $B_{12}$. Al comparar grupos, se hallaron diferencias significativas en proteínas, calcio (menor consumo en veganos) y fibra (menor en ovo-lácteo-vegetarianos). La suplementación con vitaminas y suplementos también fue muy baja. Los resultados resaltan la importancia de una evaluación constante de la ingesta de nutrientes en una dieta vegetariana, a modo de evitar deficiencias o excesos, y la insoslayable tarea del nutricionista para el asesoramiento alimentarionutricional a los individuos que expresen interés en adoptar este tipo de dieta.

Palabras clave: Dieta vegana; Dieta vegetariana; Deficiencia de proteína; Hierro en la dieta; Minerales en la dieta; Vitaminas en la dieta.

\footnotetext{
ABSTRACT

A vegetarian diet is defined by the total or partial exclusion of foods of animal origin. When properly planned it can prevent and treat disease. However, a restrictive diet in certain foods must be evaluated. The purpose of this research was
}

Meliessa Penner Teichgräf', Natalia Elizabeth González Cañete ${ }^{1 *}$.

1. Carrera de Nutrición. Facultad de Ciencias Médicas. Universidad del Pacífico. Asunción, Paraguay.

\begin{abstract}
*Dirigir la correspondencia a: Natalia González Cañete. Carrera de Nutrición Humana, Facultad de Ciencias Médicas, Universidad del Pacífico, Avda. San Martín 961 c/Avda. España, Asunción. Paraguay. Email: natalia.gonzalez580@gmail.com
\end{abstract}

Este trabajo fue recibido el 15 de abril de 2020. Aceptado con modificaciones: 25 de mayo de 2020. Aceptado para ser publicado: 26 de junio de 2020.

to describe anthropometric parameters, eating and lifestyle habits of lacto-ovo-vegetarians and vegans, and to explore differences in critical nutrient intake between both groups. We conducted a descriptive cross-sectional study, carried out among lacto-ovo-vegetarians and vegans in the city of Asunción, Paraguay, during 2015. Anthropometric parameters, intake and healthy lifestyle habits were evaluated. Seventeen lacto-ovo-vegetarians and 14 vegans participated. $67.7 \%(n=21)$ were normal weight, $29.1 \%(n=9)$ were overweight, and $3.2 \%(n=1)$ were underweight. There was an intake lower than the requirements in terms of energy, carbohydrates, proteins and fats in both groups of vegetarians, and very limited intake of calcium and vitamin $B_{12}$ in vegans. Women particularly had intake well below 
the recommendations for iron and calcium. No woman on a vegan diet met the dietary recommendation for vitamin $B_{12}$. When comparing groups, significant differences were found in terms of protein and calcium, (lower consumption in vegans), fiber (lower in lacto-ovo-vegetarians). Vitamin supplementation was also very low. The results highlight the importance of constant evaluation of nutrient intake in a vegetarian diet in order to avoid deficiencies or excesses, and the very important work of the nutritionist for food and nutritional advice for individuals who express interest in adopting this type of diet.

Keywords: Dietary iron; Dietary minerals; Dietary vitamins; Protein deficiency; Vegan diet; Vegetarian diet.

\section{INTRODUCCIÓN}

La alimentación juega un rol primordial tanto para la prevención como para el desarrollo de enfermedades. Una dieta vegetariana se define por la exclusión de carnes de todo tipo en los hábitos de alimentación de una persona ${ }^{1}$ y se basa fundamentalmente en alimentos de origen vegetal, como granos, hortalizas, legumbres, frutas, semillas y nueces ${ }^{2}$. Según el tipo de vegetarianismo del que se trate, se incluyen o no alimentos derivados de animales. Algunos tipos de dieta vegetariana son: la dieta vegana, que excluye todos los productos cárnicos y sus derivados (lácteos y huevos), y la dieta ovo-lácteo vegetariana que excluye todos los productos cárnicos, e incluye tanto huevos como lácteos ${ }^{2,3}$, entre otras. Según la Asociación Americana de Dietética (ADA), una dieta vegetariana planificada de manera adecuada puede aportar en la prevención y en el tratamiento de ciertas enfermedades ${ }^{2,4}$. Un estilo de vida vegetariano se asocia con un menor índice de masa corporal, circunferencia de cintura, colesterol total, lipoproteína de baja densidad (LDL-c) y mayor lipoproteína de alta densidad (HDL-c) $)^{5,6}$.

Según la literatura disponible, una dieta vegetariana puede llegar a cubrir el requerimiento de todos los nutrientes, siempre y cuando se realice correctamente ${ }^{1,4}$. La ingesta de nutrientes será diferente según el tipo de dieta vegetariana.

Los nutrientes críticos son aquellos cuyo déficit o exceso en la alimentación constituye un factor de riesgo. Se han reportado diferencias significativas en el consumo de energía y macronutrientes entre los vegetarianos y los no vegetarianos. El consumo de proteínas y grasas puede estar disminuido, y la ingesta de carbohidratos aumentada, al realizar comparaciones entre vegetarianos y omnívoros. En algunas investigaciones se cuestiona la ingesta adecuada de nutrientes tales como hierro, calcio, zinc, yodo, vitamina $D$ y vitamina $B_{12}$ en algunos tipos de vegetarianos, constituyéndose por lo tanto como nutrientes críticos para las personas que llevan una dieta vegetariana no planificada o equilibrada, ya que al restringir totalmente el consumo de alimentos de origen animal la ingesta de estos nutrientes podría verse disminuida ${ }^{2}$.

La última Encuesta Nacional de Alimentación y Nutrición (ENAN) del Paraguay fue realizada hace más de 50 años, y por lo tanto no se cuenta con datos representativos y actualizados que puedan dar cuenta de la frecuencia con que se presenta la dieta vegetariana a nivel nacional. Sin embargo, este tipo de dietas fue emergiendo a lo largo de los últimos años.

Considerando lo presentado anteriormente, el objetivo de este trabajo es describir parámetros antropométricos, los hábitos de alimentación y de estilo de vida en ovo-lácteovegetarianos y veganos de Asunción y Gran Asunción, Paraguay, y explorar diferencias en la ingesta de nutrientes críticos entre ambos grupos.

\section{MATERIALES Y MÉTODOS}

Estudio observacional, descriptivo, de corte transversal, con muestreo no probabilístico por bola de nieve. Se buscaron voluntarios de forma digital a través de redes sociales, durante el mes de julio del año 2015, en la ciudad de Asunción, Paraguay. Se concertó una fecha para dos encuentros con los posibles participantes. Se realizó una entrevista individual, a fin de asegurar el cumplimiento de los criterios de selección. Una vez seleccionados los participantes se realizaron las mediciones antropométricas, y se explicó la manera en que deberían registrar las variables dietéticas y de estudio. Cada participante completó un "diario de alimentos y bebidas" durante tres días (se incluyó un día no laborable de fin de semana) y una vez completado, se envió por vía electrónica a la investigadora.

\section{Participantes}

Los criterios de inclusión fueron: ser ovo-lácteovegetariano o vegano, con una edad entre 19 y 59 años, con residencia en Asunción y Central. Se excluyó a embarazadas o en período de lactancia, y a individuos con alguna patología diagnosticada que requiriera dietoterapia (gastroenteritis aguda, o patología gastrointestinal crónica como: celiaquía, sensibilidad al gluten no celiaca, síndrome de intestino irritable, enfermedad de Crohn, insuficiencia renal aguda y crónica, enfermedades hepáticas, errores innatos del metabolismo, etc.).

\section{Variables}

Las variables descriptivas fueron edad y sexo. Las variables antropométricas que se incluyeron fueron: estado nutricional por IMC, circunferencia abdominal y masa grasa. Las variables alimentarias-nutricionales fueron: tipo de dieta vegetariana (dieta ovo-lácteo-vegetariana o vegana), duración de la dieta vegetariana (años), consumo esporádico de alimentos no aceptados en estas dietas, motivos por los cuales seguían una dieta vegetariana, tipo de dieta anterior a la adopción de la dieta actual, tipo de suplementos dietarios y bebidas consumidas diariamente, adecuación de las recomendaciones dietarias de consumo de macronutrientes, fibra y nutrientes críticos (para los fines de esta investigación se consideraron como nutrientes críticos al calcio, hierro y vitamina $B_{12}$ ) y requerimientos de energía. Las variables relacionadas al estilo de vida fueron: consumo actual o histórico de cigarrillos y de alcohol, y nivel de actividad física. 


\section{Tamaño de muestra}

Dado que durante el periodo en el que se desarrolló el estudio (año 2015) la dieta vegetariana no era una elección común de alimentación en la población, y dado que el muestreo sería no probabilístico, se decidió incluir a todas las personas dispuestas a participar, y que se hayan encontrado durante el periodo de recolección de datos.

\section{Procedimientos y técnicas}

Se realizó una entrevista a cada participante, obteniéndose información de la historia alimentaria y de estilo de vida. Para determinar el estado nutricional se pesó y midió a los sujetos, utilizándose un tallímetro de aluminio plegable Nutrisys $^{\circledR}$ (origen Paraguay), con una cinta métrica Seca ${ }^{\circledR}$ (origen Alemania), modelo 201 con capacidad de 2,05 m y precisión de $0,1 \mathrm{~mm}$, incorporada al tallímetro. El peso fue medido con la balanza portátil UFESA ${ }^{\circledR}$ (origen Uruguay) con precisión de $100 \mathrm{~g}$ para el peso y capacidad de $150 \mathrm{~kg}$. Se siguieron las técnicas convencionales de medición para peso y talla ${ }^{7}$. El estado nutricional se definió según la clasificación del Índice de Masa Corporal (IMC) ${ }^{7}$. Para la determinación del porcentaje de masa grasa se midieron cuatro pliegues cutáneos (bicipital, tricipital, subescapular y supra iliaco) siguiendo la metodología ISAK, utilizando un plicómetro de plástico Slim Guide ${ }^{\circledR}$ (origen EE.UU.) con capacidad de 0 a $85 \mathrm{~mm}$ y precisión de $1 \mathrm{~mm}$. Se utilizó la ecuación de densidad corporal de Durnin-Womersley ${ }^{8}$ para aplicarla en las ecuaciones de \% de masa grasa de Siri ${ }^{8}$. Se consideró como un alto porcentaje de grasa a $>20 \%$ para hombres y $>33 \%$, para mujeres de 20 a 39 años de edad, $y>22$ para hombres y $>34 \%$ para mujeres de 40 a 59 años de edad 9 . La circunferencia abdominal fue medida en el punto medio entre la cresta iliaca y el último reborde costal, utilizándose una cinta métrica de metal inextensible Lufkin Executive ${ }^{\circledR}$ (origen EE.UU.), con capacidad de $2 \mathrm{~m}$ y precisión de 0,1 $\mathrm{mm}$. Se consideró como presencia de riesgo cardiovascular a un valor $\geq$ a $94 \mathrm{~cm}$ para hombres $\mathrm{y} \geq \mathrm{a} 80 \mathrm{~cm}$ para mujeres (7). Los participantes completaron en sus hogares un registro diario de alimentos y bebidas por tres días, en un formato estandarizado por la investigadora, incluyendo un día no laborable. Durante el primer encuentro entre la investigadora y los participantes se proporcionaron indicaciones verbales para registrar los alimentos de manera completa, incluyendo marcas, tipo o versión del alimento o producto alimenticio, etc., aunque también se entregaron indicaciones por escrito, que ayudarían principalmente a estimar las porciones y luego la cantidad de alimentos. Estas indicaciones incluían imágenes fotográficas de porciones estándar de alimentos, con objetos de uso común al lado de los alimentos para facilitar la estimación, como pelotas de tenis, juegos de naipes, $\mathrm{cd}^{\prime} \mathrm{s}$, etc ${ }^{10,11}$. Para determinar la cantidad de consumo de alimentos, se ofrecieron tres posibilidades a los encuestados: pesado en balanzas de alimentos de uso casero (balanzas estandarizadas por la investigadora), estimación de las porciones consumidas mediante las imágenes fotográficas estándar de porciones (entregadas en la guía), o envío de fotografías a la investigadora (vía celular) con porciones de alimentos consumidos, siempre al lado de un objeto de tamaño estándar y fijo, que permitiera su interpretación posterior (por ejemplo, fotografía de lámina de queso al lado de una cédula de identidad), y con suficiente luz en la fotografía para observar detalles. Podían elegir cualquiera de las tres opciones simultáneamente. En caso de que los sujetos de estudio enviaran fotografías, la investigadora posteriormente debía por su cuenta buscar un alimento de la misma marca o tipo y pesarlo, de acuerdo al tamaño visualizado en la fotografía. Cabe aclarar que el consumo de bebidas también fue contemplado para el análisis de nutrientes. Los datos obtenidos del registro alimentario fueron analizados mediante el software Nutrimind 2012 (México). Este software incluye bases de datos de composición química de alimentos de la United States Department of Agriculture (USDA). Para el cálculo del Valor Calórico Total (VCT) consumido por los sujetos se multiplicó el consumo de los g de carbohidratos y de proteínas por $4(\mathrm{kcal})$, y el consumo de g de grasas por 9 (kcal). Para estimar el requerimiento de energía de los sujetos, se utilizaron las ecuaciones de la FAO/OMS (2001) ${ }^{12}$. La actividad física del individuo fue estimada mediante una entrevista, empleando la versión corta del Cuestionario Internacional de Actividad Física (IPAQ) ${ }^{13}$. Para la clasificación de la AF en baja, moderada o alta, se consideró la interpretación explicada en la literatura ${ }^{14}$ que considera los Mets (Metabolic Equivalent of Task o Unidades de Índice Metabólico) por minuto y semana. Para obtener el número de Mets se multiplicó cada uno de los valores (3,3 para caminatas, 4 para actividad moderada u 8 para actividad vigorosa) por el tiempo en minutos de la realización de la actividad en un día y por el número de días a la semana que se realiza. El cuestionario IPAQ incorpora una serie de imágenes que ayuda al encuestado a identificar si las actividades que realizó corresponden a actividades vigorosas o moderadas ${ }^{14}$.

Para la determinación del requerimiento de nutrientes se utilizó como referencia la Ingesta Adecuada (IA) de fibra, y la Recomendación dietaria (RD) para calcio $^{15}$, hierro $^{16}$ y vitamina $\mathrm{B}_{12}{ }^{17}$. Para el cálculo del porcentaje de adecuación de la ingesta de kcal, el valor del VCT consumido se multiplicó por 100, y éste resultado se dividió por el requerimiento de energía. El cálculo de requerimientos y porcentaje de adecuación de los macronutrientes se realizó siguiendo las recomendaciones actuales de consumo de macronutrientes ${ }^{18}$ :

- Requerimiento de proteínas: Se utilizó un aporte proteico de $0,8 \mathrm{~g} / \mathrm{kg}$ de peso real. Se realizó la conversión de este valor en kcal y en porcentaje según el VCT requerido. Para el \% de adecuación, los g de proteínas ingeridos por el sujeto se multiplicaron por 100, y este resultado se dividió por los g de proteínas calculados como requeridos.

- Requerimiento de lípidos: Se consideró un 30\% del VCT requerido. Para el \% de adecuación, los g de lípidos ingeridos por el sujeto se multiplicaron por 100, y este resultado se dividió por los g de lípidos calculados como requeridos. 
- Requerimiento de Carbohidratos (CHO): se obtuvo por diferencia de porcentaje: $100 \%$ - (\% de proteínas) - (\% de grasa $)=\%$ de carbohidratos. Se realizó la conversión de este valor en kcal y g. Para el \% de adecuación, los $\mathrm{g}$ de $\mathrm{CHO}$ consumidos por el sujeto se multiplicaron por 100

, y este resultado se dividió por los g requeridos. Para los tres macronutrientes, se consideró que si el porcentaje obtenido resultaba $<90 \%$ en relación al requerimiento estimado se consideraba como una dieta insuficiente, $>110 \%$ dieta con un consumo mayor al requerimiento, y entre 90 y $110 \%$ una dieta con un consumo adecuado del nutriente. Para la determinación del \% de adecuación de fibra, calcio, hierro y vitamina $B_{12}$ se empleó una regla de tres simple, utilizando el valor de la ingesta de micronutrientes (en g, mg o $\mu$ g) y considerando como el $100 \%$ el valor de la recomendación dietaria (RD) o ingesta adecuada (IA) para la edad y el sexo. Si el porcentaje de adecuación resultaba $<90 \%$ en relación al RD o IA se consideraba como una dieta insuficiente en cuanto al nutriente en cuestión, $>110 \%$ dieta con un consumo mayor a la RD e IA del nutriente, y entre 90 y $110 \%$ una dieta con un consumo adecuado del nutriente ${ }^{7}$. Para comparar la ingesta de ciertos nutrientes como grupo se utilizó el Requerimiento Promedio Estimado (RPE) que se considera como las cantidades de un nutriente que cubrirían los requerimiento del $50 \%$ de una población.

El protocolo fue aprobado por el Comité de Ética de la Universidad del Pacífico, cumpliéndose las normas éticas de la Declaración de Helsinki de 1975, con revisión de octubre de 2000. Se obtuvo el consentimiento informado de los participantes. No se presentaron conflictos de interés.

\section{Análisis estadístico}

Los datos fueron digitalizados en planilla electrónica de Microsoft Office Excel ${ }^{\circledR}$ Versión 2010 (EE.UU.) y analizados con el programa estadístico Social Science Statistics. Las variables cuantitativas se expresaron como mediana y percentil 25 y 75, y las cualitativas con frecuencias y porcentajes. Para explorar la presencia de diferencias significativas entre los dos grupos de vegetarianos se utilizó el Test de WilcoxonMann-Whitney. Se consideró una diferencia significativa a un valor $p$ menor que 0,05 .

\section{RESULTADOS}

Participaron un total de 31 sujetos: 17 ovo-lácteovegetarianos y 14 veganos. Ninguno de los participantes abandonó el estudio durante el periodo de relevamiento de datos. La mayoría de las personas residían en Asunción $(n=20)$, y el resto en localidades cercanas a Asunción: cuatro en Luque, tres en Fernando de la Mora, uno en Lambaré, uno en Mariano Roque Alonso, uno en Ñemby y otro en San Lorenzo. La mediana de tiempo que llevaban siguiendo la dieta fue de 4 y 1,1 años, para ovo-lácteo-vegetarianos y veganos, respectivamente. En 6 participantes $(25,8 \%)$ se encontró un nivel alto de masa grasa corporal. Ningún sujeto presentó un nivel muy alto de masa grasa corporal, sólo en dos (6,5\%) se encontró baja masa grasa según sexo y edad (Tabla 1). En cuanto al riesgo cardiovascular, 10/17 $(58,8 \%)$ y $11 / 14(78,6 \%)$ de los ovo-lácteo-vegetarianos y veganos respectivamente no presentaron riesgo; 5/17 (29,4\%) y $3 / 14(21,4 \%)$ de los ovo-lácteo-vegetarianos y veganos presentaron riesgo aumentado, y $2 / 17$ (11,8\%) de los ovolácteo-vegetarianos presentaron riesgo muy aumentado.

La ingesta de energía, carbohidratos, proteínas y lípidos fue insuficiente con respecto a los requerimientos

Tabla 1. Variables demográficas y antropométricas de los participantes del estudio según tipo de dieta vegetariana.

\begin{tabular}{|lcc|}
\hline Variables & Ovo-lácteo-vegetarianos & Veganos \\
& $\begin{array}{c}\text { Total } \\
(\mathbf{n = 1 7})\end{array}$ & $\begin{array}{c}\text { Total } \\
(\mathbf{n}=\mathbf{1 4})\end{array}$ \\
& $24(23-31)$ & $30,5(23,2-40)$ \\
& $68,8(58,7-78,0)$ & $56,2(52,6-65,0)$ \\
Edad (años) & $1,67(1,64-1,72)$ & $1,62(1,60-1,69)$ \\
Talla $(\mathrm{m})$ & $24,2(21,8-26,2)$ & $20,5(19,6-23,4)$ \\
IMC $\left(\mathrm{kg} / \mathrm{m}^{2}\right)$ & $87,5(80,7-90,7)$ & $77,5(74,3-82)$ \\
Circunferencia abdominal $(\mathrm{cm})$ & $25,4(20-28,7)$ & $25,4(19,3-30,2)$ \\
Masa grasa $(\%)$ & & \\
& & \\
\hline
\end{tabular}

IMC: Índice de Masa Corporal.

Los resultados se presentan como percentil 50 (p25-p75). 
en ambos grupos de vegetarianos (Figuras 1 y 2). En cuanto a las vitaminas y minerales, la ingesta fue insuficiente para calcio y vitamina $B_{12}$ en ambos grupos, e insuficiente para hierro en los veganos. Los mismos superaron el \% de adecuación para fibra de manera grupal (Figura 2). Al analizar los requerimientos de manera individual, ninguno de los varones ovo-lácteo-vegetarianos cumplió con la IA de fibra (38 g/día). La IA de fibra para las mujeres es de 25 g/día, recomendación que se cumplió en 8/17 (44,4\%) de las ovo-lácteo-vegetarianas y en 11/14 (81,8\%) de las veganas (Tabla 2). Con respecto al consumo de calcio, en los varones veganos la mediana de la ingesta de calcio fue de $434 \mathrm{mg} /$ día. Sólo un vegano cumplió con la RD de calcio. La RD de 1000 mg se cumplió en 4/8 (50\%) de los varones ovo-lácteo-vegetarianos (Figura 1 y Tabla 2). Al realizar la comparación de los mg de calcio consumidos por ambos grupos de varones con el valor de $800 \mathrm{mg}$ (el cual representa el RPE de calcio para hombres), la ingesta de calcio de los varones no difirió significativamente en relación al RPE, es decir, aparentemente, el consumo de calcio no fue significativamente distinto a las cantidades necesarias para cubrir el requerimiento de calcio del $50 \%$ de una población de dicho sexo. Con respecto a las mujeres, en cuanto a la RD de calcio, 4/9 (44,4\%) de las ovo-lácteovegetarianas cumplió con ella, mientras que ninguna vegana la cumplió (Figuras 1 y 2). Al realizar la comparación de la ingesta con el RPE de 1000 mg (RPE para mujeres), la ingesta de calcio de las veganas difirió significativamente con el RPE $(p=0,003)$.

Con respecto a la ingesta de hierro, 23/31 (75\%) de los vegetarianos de ambos grupos cumplió con la RD de hierro (8 mg/día). Al realizar la comparación de los mg de hierro consumidos con $6 \mathrm{mg}$ (RPE de hierro para varones), la ingesta de hierro de los varones ovo-lácteo-vegetarianos difirió significativamente con el RPE $(p=0,01)$. Es decir, la

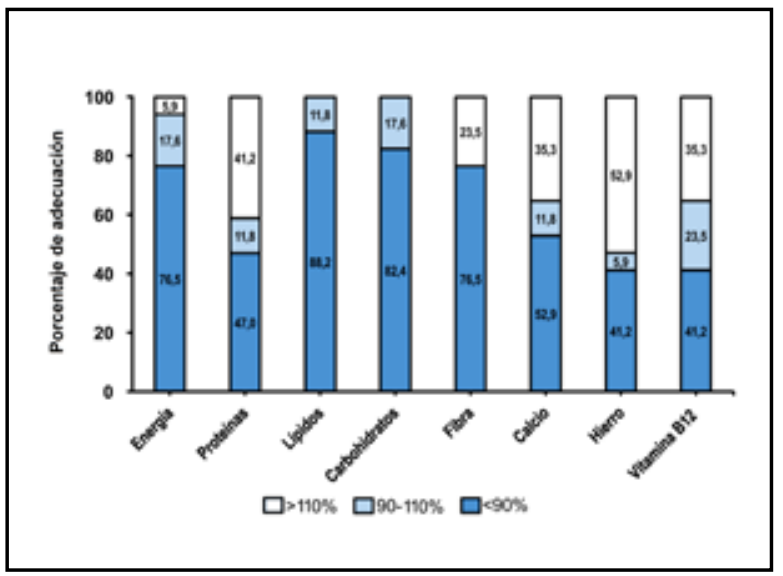

Figura 1: Porcentaje de individuos con dieta ovo-lácteovegetariana, con cumplimiento de los niveles de adecuación de energía, macronutrientes, fibra, y nutrientes críticos. mayoría tuvo una ingesta significativamente superior a la RD de hierro. La ingesta de hierro de los veganos no fue significativamente distinta al RPE. En el caso de las mujeres, la RD del hierro es de $18 \mathrm{mg} / \mathrm{d}$ áa, valor que no se cumplió en ninguno de los dos grupos. Al realizar la comparación de los mg de hierro consumidos con 8,1 mg (RPE de hierro para mujeres), la ingesta de hierro en ambos grupos de mujeres no difirió significativamente en relación al RPE.

Por último, en cuanto a la vitamina $\mathrm{B}_{12}, 6 / 8(75 \%)$ varones ovo-lácteo-vegetarianos cumplió con la RD de la vitamina $(2,4 \mathrm{mg} /$ día). Al realizar la comparación de los $\mu \mathrm{g}$ de vitamina $B_{12}$ consumidos con 2,4 $\mu$ g (RPE para los varones), la ingesta no difirió significativamente en ninguno de los grupos de vegetarianos. La RD de vitamina $B_{12}$ para mujeres es de $2,4 \mu \mathrm{g} / \mathrm{d}$ áa, recomendación que se cumplió en $4 / 9(44,4 \%)$ de las ovo-lácteo-vegetarianas. Ninguna vegana cumplió con la RD de vitamina $\mathrm{B}_{12}$ (Figura 2). Al realizar la comparación de los $\mu \mathrm{g}$ de vitamina $\mathrm{B}_{12}$ consumidos con el RPE (de 2,4 $\mu$ g para mujeres), la ingesta de vitamina $B_{12}$ de las VE difirió significativamente con el RPE $(p=0,04)$, mientras que en las ovo-lácteo-vegetarianas no difirió significativamente. Los valores resumen de la cantidad ingerida de energía y nutrientes según tipo de vegetarianismo se presenta en la tabla 2.

Se exploró la presencia de diferencias significativas en algunas variables de ingesta entre ambos grupos de vegetarianos (Tabla 2). Se hallaron diferencias significativas en el consumo de proteínas $(p=0,01)$ y calcio $(p=0,0006)$, siendo significativamente menor en el grupo de veganos. Lo mismo sucedió con la fibra $(p=0,019)$, siendo significativamente menor en los ovo-lácteo-vegetarianos. También se encontró diferencia significativa en la ingesta de vitamina $B_{12}$ según el tipo de dieta vegetariana $(p=0,002)$.

Las razones por las que los sujetos refirieron iniciar y seguir una dieta vegetariana fueron múltiples: $24 / 31$ (77,4\%)

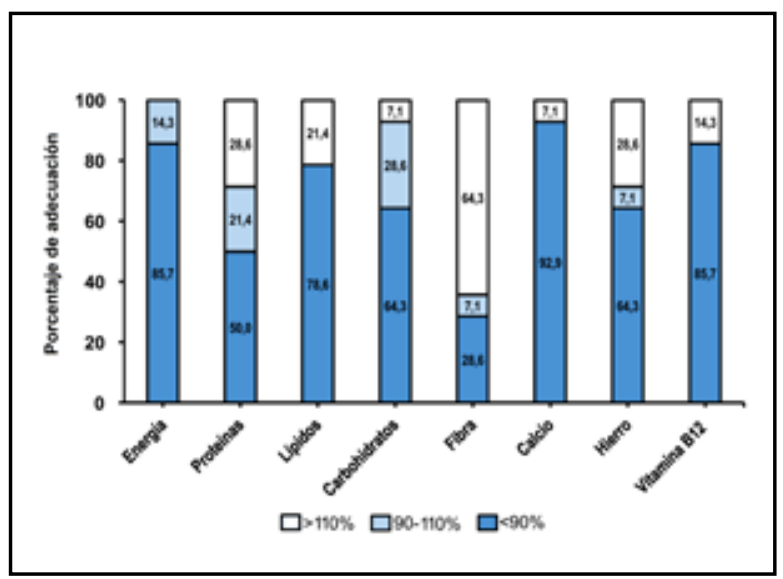

Figura 2: Porcentaje de individuos con dieta vegana, con cumplimiento de los niveles de adecuación de energía, macronutrientes, fibra, y nutrientes críticos. 
Tabla 2. Ingesta de energía, macronutrientes, fibra, y nutrientes críticos de los participantes del estudio, según tipo de dieta vegetariana.

\begin{tabular}{|c|c|c|c|}
\hline Variables & $\begin{array}{c}\text { Ovo-lácteo-vegetarianos } \\
\text { Total }(n=17)\end{array}$ & $\begin{array}{c}\text { Veganos } \\
\text { Total }(n=14\end{array}$ & Valor $p$ \\
\hline Energía (kcal/día) & $1739(1421,4-2111)$ & $1696,3(1249,4-1923,2)$ & 0,691 \\
\hline Aporte calórico (kcal/kg/día) & $25,7(18,9-29,9)$ & $29,2(21,1-36,2)$ & 0,218 \\
\hline Aporte proteico (g/kg/día) & $0,70(0,55-1,00)$ & $0,80(0,60-1,00)$ & 0,289 \\
\hline Proteínas (g/día) & $53(37,8-76,3)$ & $38(30,2-49,5)$ & 0,011 \\
\hline Lípidos (g/día) & $46(34,9-70,5)$ & $47,8(32,5-59,1)$ & 0,811 \\
\hline CHO (g/día) & $243,6(206,6-316,9)$ & $296,1(186,6-320,9)$ & 0,866 \\
\hline Fibra (g/día) & $19,9(13,9-30,1)$ & $31,3(26,7-38,4)$ & 0,020 \\
\hline Calcio (mg/día) & $845(558,8-1355)$ & $430,3(287,4-520,8)$ & $<0,05$ \\
\hline Hierro (mg/día) & $12,3(8,8-16,6)$ & $11,3(8,1-18,4)$ & 0,662 \\
\hline Vitamina $B_{12}(\mu g / d i ́ a)$ & $2,3(1,1-3,6)$ & $0(0-1,0)$ & $<0,05$ \\
\hline
\end{tabular}

CHO: carbohidratos. Los resultados se presentan como Mediana (p25-p75). Valor p según Test de Wilcoxon-Mann-Whitney.

por respeto a la vida animal-principios animalistas, 19/31 $(61,3 \%)$ por beneficios a la salud, $7 / 31(22,6 \%)$ por filosofía de vida-espiritualidad, 5/31 (16,1\%) por cuidado al medio ambiente, y 8/31 (25,8\%) por otros motivos como religión, ética. El tipo de dieta vegetariana que seguían ambos grupos de vegetarianos antes de su plan alimentario actual fue mayoritariamente omnívora en 16/31 (51,6\%). En el caso de los veganos $9 / 14(64,3 \%)$ siguieron previamente una dieta ovo-lácteo-vegetariana, y en 1/14 (7,1\%) omnívora. Sólo en el grupo de veganos se presentaron dietas de tipo lacto-vegetariana en 2/14 (14,3\%), ovo-vegetariana en $1 / 14(7,1 \%)$ y pesco-ovo-vegetariana $1 / 14(7,1 \%)$ antes de llevar el plan alimentario actual. En 3/31 (10\%) de los sujetos se producía el consumo esporádico de carne. En cuanto a los veganos, 4/14 (28,5\%) y 7/14 (50\%) consumían esporádicamente lácteos/huevos, y miel, respectivamente. Con respecto a la suplementación sólo 2 veganos (14\%) consumían suplementos: uno de ellos de vitaminas $B_{12}$ $B_{9}$ y espirulina, y el otro sujeto un multivitamínico, batido proteico, suplemento de ácido graso y de aminoácidos. En el caso de los ovo-lácteo-vegetarianos, dos sujetos $(11,7 \%)$ consumieron multivitamínicos, dos batidos proteicos y uno suplemento de algas; entre los veganos dos consumieron multivitamínicos, uno batidos proteicos, uno suplemento de algas y uno suplemento de ácidos grasos. Diariamente, $30 / 31(96,7 \%)$ refirió consumir agua, 14/31 (45,1\%) jugos naturales, 4/31 (12,9\%) jugos artificiales, 6/31 (19,3\%) bebidas derivadas de semillas, $11 / 31$ (35,4\%) café, té, mate, tereré (el $50 \%$ de los veganos referían consumirlo diariamente), y $2 / 31(6,4 \%)$ kéfir.
En $10 / 17(58,8 \%)$ y en $9 / 14(64,1 \%)$ de los ovolácteo-vegetarianos y veganos se presentó $\mathrm{AF}$ moderada, respectivamente. En 6/17 (35,3\%) ovo-lácteo-vegetarianos, y en $4 / 14(28,6 \%)$ veganos se presentó AF baja, y en 1/17 $(5,9 \%)$ ovo-lácteo-vegetariano y $1 / 14(7,1 \%)$ vegano se presentó AF alta. El $100 \%$ de los participantes no fumaba al momento de la realización del estudio. Fumaron en el pasado $11 / 31$ (35,5\%) sujetos, mientras que $17 / 31$ (54,5\%) de los participantes refirió haber dejado de fumar al iniciar la alimentación vegetariana. Con respecto al consumo de alcohol, 13/31 (42\%) refirió consumir alcohol habitualmente, 16/31 (50\%) refirió haber consumido alcohol anteriormente y de este grupo $7 / 16$ (44,4\%) dejó de consumir alcohol al comenzar la dieta vegetariana. Ningún individuo refirió consumir alcohol de manera diaria.

\section{DISCUSIÓN}

Los hallazgos de IMC de algunos estudios concordaron con los del presente estudio (veganos con valores de IMC más bajos que otros vegetarianos) ${ }^{4,19}$. Todas las variables antropométricas (peso, IMC, circunferencia abdominal y porcentaje de masa grasa) se presentaron con valores menores en los veganos, en comparación con los ovo-lácteovegetarianos. Clarys et $\mathrm{al}^{20}$, en un estudio realizado en 1320 vegetarianos, la proporción de veganos $(\mathrm{n}=104)$ eutróficos fue de $78,8 \%$, y de ovo-lácteo-vegetarianos (n= 573) $72,9 \%$. El $1,9 \%$ de los veganos se encontró con obesidad, a diferencia del 3,5\% de los ovo-lácteo-vegetarianos. En el referido estudio $^{20}$, la mayoría se encontró con un nivel adecuado de \% de masa grasa, además de presentar una ingesta de 
energía menor en los veganos, en comparación con los ovolácteo-vegetarianos, semi-vegetarianos, pesco-vegetarianos y omnívoros. Este hallazgo coincide con el presente estudio, en el cual la mayoría de los participantes presentó un consumo de energía $(\mathrm{kcal})$ menor a los requerimientos. Los alimentos de origen animal son densos en energía debido al contenido en proteína, grasa, colesterol y pueden contribuir al aumento del VCT de un individuo. Sin embargo, esto no significa que una dieta que excluye totalmente los alimentos de origen animal resulte necesariamente baja en kcal. Así lo demuestra el estudio de Rizzo et $\mathrm{a}^{21}{ }^{21}$, realizado en 71751 vegetarianos, en el cual fueron los semi-vegetarianos $(\mathrm{n}=$ 4042), considerados como todos aquellos que consumían de forma esporádica carnes, huevos y lácteos $\geq 1 \mathrm{vez} /$ mes, pero menos que 1 vez/semana) quienes tuvieron un menor consumo de energía, y no los pesco-vegetarianos $(n=6583)$, ovo-lácteo-vegetarianos $(n=21799)$, o veganos $(n=5694)$. En el presente estudio se distinguió un mayor consumo de proteínas en los ovo-lácteo-vegetarianos, pero un mayor aporte proteico en los veganos. Esto podría explicarse por el menor peso que presentaron los veganos. Se debe tener en cuenta que, aunque el grupo de veganos cumpla con el aporte proteico mínimo de $0,8 \mathrm{~g} / \mathrm{kg}$, al ser las proteínas exclusivamente de origen vegetal, la calidad proteica es diferente. Una persona puede tener un consumo adecuado en cantidad de proteínas, pero deficiente en su calidad. La calidad de una proteína depende del aporte de aminoácidos y su biodisponibilidad. Según un estudio realizado por Suárez López et $\mathrm{a}^{21}$, los alimentos de origen animal no presentaron ningún aminoácido limitante. La soja, el garbanzo, el pistacho y la remolacha fueron los únicos alimentos de origen vegetal que no presentaron aminoácidos limitantes. Debido a la menor digestibilidad de las proteínas no hemínicas, la ADA refiere que las necesidades proteicas podrían ser mayores en individuos cuyas fuentes dietéticas de proteína son aquellas que son digeridas en menor eficiencia, tales como algunos cereales (bajos en lisina) y legumbres (bajos en metionina, cistina y triptófano) $)^{2}$.

Los ovo-lácteo-vegetarianos presentaron mayoritariamente un consumo insuficiente de fibra, mientras que los veganos superaron ampliamente el consumo. Este resultado concuerda con estudios en los cuales también fueron los veganos quienes presentaron mayor consumo de fibra en comparación con los ovo-lácteo-vegetarianos, pescovegetarianos y semi-vegetarianos ${ }^{22}$. Debe llamar la atención las cantidades de fibra consumida por los veganos, dado que los alimentos ricos en fibra son también ricos en anti-nutrientes (como el ácido fítico), que condicionan la absorción de minerales críticos para esta población, como el hierro. Con respecto al mismo, la RD se cubrió en los varones de ambos grupos, no así en las mujeres. Ninguna de las mujeres ovo-lácteo-vegetarianas o veganas cumplió con la RD de hierro. Las mujeres en edad fértil son las más propensas a presentar deficiencia de hierro, y mantienen altos requerimientos hasta la menopausia ${ }^{23}$. En la población estudiada se estaría dando una situación desfavorecedora para la absorción de hierro, la cual sería muy alta ingesta de fibra, e incumplimiento de los requerimientos de hierro, sobre todo en veganas. Dado que el hierro no hem es el único tipo de hierro presente en las dietas veganas, debe enseñarse a los vegetarianos acerca de los factores que limitan su absorción ${ }^{24,25,26}$. Un estudio que involucró a ovo-lácteo-vegetarianos y veganos españoles describió la ausencia de diferencias en el estado de hierro entre ambos grupos, sin influencias dadas por la duración de la dieta. Los hombres presentaron suficiente hierro, mientras que la deficiencia fue frecuente en mujeres (ferritina $\geq 15$ a $\leq 30 \mathrm{ng} / \mathrm{mL}$ ), por lo que se recomendaba que las mismas aplicaran estrategias para aumentar su biodisponibilidad, especialmente ante las pérdidas intensas de sangre durante la menstruación ${ }^{27}$. En la presente investigación, la minoría de los sujetos consumía suplementos vitamínicos y/o minerales, situación que debería llamar la atención por la implicancia que la suplementación tiene sobre las reservas de minerales, especialmente en veganos. Muchos vegetarianos prefieren evitar los alimentos industrializados, rechazando por este motivo la opción de suplementación, u optando por suplementos de origen natural ${ }^{28}$. El consumo de alimentos fortificados sería en este caso fundamental para obtener una ingesta adecuada de micro-nutrientes ${ }^{4}$.

La ingesta adecuada de calcio en los veganos es también a menudo una preocupación. En este estudio las veganas tuvieron el menor consumo de calcio, no alcanzándose siquiera el $50 \%$ de la RD. La ingesta inadecuada de calcio y vitamina $\mathrm{D}$ puede provocar una disminución en la densidad mineral ósea, especialmente en dietas que restringen el consumo de lácteos, o combinan la baja ingesta de calcio con un alto consumo de fibra (situación que se dio en la población estudiada) ${ }^{27}$. En personas veganas el riesgo de fracturas es mayor que el riesgo encontrado en personas que incluyen lácteos en su alimentación ${ }^{29}$.

Los veganos del presente estudio presentaron un consumo muy deficiente de vitamina $B_{12}$. En caso de los ovo-lácteovegetarianos los resultados indicaron un consumo adecuado, dado posiblemente por el mayor consumo de alimentos de origen animal. Resultados similares se encontraron en el estudio de Rizzo et $\mathrm{al}^{22}$. En dicho estudio hallaron un mayor consumo de la vitamina $B_{12}$ en los pesco-vegetarianos y semi-vegetarianos. En un estudio de cohorte prospectivo realizado en Polonia, 20 personas anteriormente omnívoras siguieron una dieta vegana por 5 años. Diez de ellas basaron su dieta en alimentos naturales, mientras que las otras 10 basaron su dieta en alimentos fortificados con vitamina $B_{12}$. En el grupo que consumió alimentos fortificados el nivel plasmático de vitamina $\mathrm{B}_{12}$ se presentó dentro del rango adecuado, mientras que en dos sujetos que consumieron alimentos naturales los niveles se encontraron fuera del rango. En ambos grupos la concentración sérica de la vitamina $\mathrm{B}_{12}$ disminuyó de manera significativa luego de cinco años de haberse iniciado la transición de una dieta omnívora a vegana $^{30}$. Algunos investigadores refieren la importancia 
de re-definir los valores plasmáticos adecuados de esta vitamina, debido a que se observan efectos negativos (como neuropatías) aún con una concentración sérica de vitamina $\mathrm{B}_{12}$ dentro del rango. La deficiencia metabólica a largo plazo de esta vitamina se asocia con defectos del tubo neural, déficit cognitivo, atrofia del cerebro, depresión, neuropatía y enfermedad de Alzheimer ${ }^{31}$. La suplementación de $B_{12}$ es fundamental, en particular para los veganos: una investigación realizada en tres grupos de alemanes: corredores recreacionales (omnívoros, ovo-lácteo-vegetarianos y veganos) describió que cerca del $80 \%$ de cada grupo mostró adecuación de vitamina $\mathrm{B}_{12}$, con niveles más altos en usuarios que consumían suplementos ${ }^{32}$. Un estudio realizado en España con adultos ovo-lácteo-vegetarianos y veganos, detectó deficiencia subclínica de vitamina $\mathrm{B}_{12}$ (determinado mediante el ácido metilmalónico sérico) en $>10 \%$ de los vegetarianos, mientras que $>70 \%$ de los participantes mostró hiperhomocisteinemia (Hcy>15 $\mu \mathrm{mol} / \mathrm{L}$ ). Se observó una Hcy significativamente mayor en los ovo-lácteo-vegetarianos en comparación con los veganos $(\mathrm{p}=0,019)$. Además, el uso de suplementos de vitamina $B_{12}$ implicó una mejora del estado de esta vitamina, aumentando además el folato de eritrocitos $(p=0,024)$. Los investigadores señalan que es la suplementación de $\mathrm{B}_{12}$, más que las fuentes dietéticas, las que influyen de mayor manera sobre los marcadores funcionales de esta vitamina ${ }^{33}$.

Analizando los datos cuantitativos de ingesta, resulta interesante asociar los resultados de la presente investigación con los hallados en un estudio realizado también con vegetarianos de Paraguay, en el año 2017, entre cuyos objetivos se encontró evaluar el conocimiento con respecto a nutrientes críticos y posibles consecuencias de su bajo aporte. Participaron 132 vegetarianos: el 41\% presentó un nivel de conocimiento insuficiente con respecto a los nutrientes críticos, un 30,3\% conocimientos suficientes, y un $29 \%$ conocimiento regular ${ }^{34}$. El respeto animal, las razones filosóficas y espirituales, la religión y los beneficios para la salud son algunos de los motivos referidos por los sujetos de esta investigación para practicar el vegetarianismo. En el estudio referido, también realizado en Paraguay, los principales motivos para seguir el vegetarianismo fueron también los principios animalistas (50,8\%), beneficios para la salud $(21,2 \%)$ y creencias religiosas/motivos ecológicos $(9,1 \%)^{34}$. Un estudio realizado en Chile también describió al principio animalista como principal motivo para seguir el vegetarianismo. Según ésta investigación, la deficiencia de nutrientes es mayor en personas que deciden ser vegetarianos por motivos intelectuales (éticos, morales, espirituales, religiosos) y sociales (económicos, ecológicos, políticos) que en personas que deciden ser vegetarianos por razones de salud ${ }^{35}$.

Con respecto al consumo de bebidas, debe recordarse que los taninos presentes en el té y café reducen la biodisponibilidad del hierro. Estas bebidas deben consumirse alejadas de hortalizas, cereales y lácteos para evitar la interferencia en la absorción del hierro ${ }^{26,36,37}$. En el presente estudio, el 50\% de los veganos refirieron consumir café, té, mate, tereré de manera diaria, por lo que sería importante proporcionar pautas de consumo para evitar la reducción en la absorción de hierro. El consumo de lácteos también debe estar alejado del consumo de hortalizas y cereales integrales, para evitar la formación de sustancias que disminuyan la absorción de calcio, como el oxalato o fitato de calcio ${ }^{26}$. El estudio de Rizzo et $\mathrm{a}^{22}$, y el estudio realizado por Tantamango-Bartley et a ${ }^{38}$ también describieron aspectos del estilo de vida según diferentes patrones dietéticos. El menor porcentaje de individuos que fumaban y consumían alcohol al momento de la realización de dichos estudios correspondía a los ovo-lácteo-vegetarianos y veganos, en contraste con los pesco-vegetarianos, semi-vegetarianos y no vegetarianos ${ }^{22}$. Ninguno de los participantes fumaba al momento de la realización del estudio, lo que resulta positivo pues no se alterarían los requerimientos de ciertas vitaminas. La actividad física (AF) realizada por los vegetarianos fue mayoritariamente moderada en ambos grupos. Esto es importante ya que una alimentación saludable conjuntamente con la realización de AF disminuye el riesgo de morbimortalidad por diferentes causas ${ }^{39}$.

Con respecto a las limitaciones de este estudio, es necesario aclarar que si bien el número de sujetos por grupo fue casi similar (17 ovo-lácteo-vegetarianos y 14 veganos), la cantidad de varones y mujeres por grupo sí fue muy distinta, existiendo mayor cantidad de mujeres en el grupo de veganas. El tamaño de la muestra de varones o mujeres por grupo no posibilita la determinación de diferencias entre sexos o entre algunas variables nutricionales. La exploración de diferencias significativas se dio entre los grupos totales. La ausencia de diferencias significativas en algunas variables nutricionales y de diferencias en relación al RPE, si las hubiere (como la ingesta de energía, proteínas, lípidos, $\mathrm{CHO}$ e hierro) podría haberse dado por no alcanzarse una $n$ suficiente para demostrar diferencias. Otra limitación fue el sesgo presente por las distintas marcas de balanzas utilizadas por los sujetos (si bien la investigadora evaluó que todas midieran lo mismo con un peso patrón) y por diferencias en la estimación del tamaño de porciones en los casos en que no se contó con las balanzas (por más que se los haya instruido brevemente previo al inicio de la investigación y se les haya entregado una guía con imágenes para facilitar la estimación). Entre otras limitaciones presentadas se menciona también la posibilidad de un sesgo dada la forma en que se seleccionó a los participantes (mediante redes sociales, sesgo de selección), o a la presencia del sesgo de deseabilidad social (fenómeno que hace que los individuos se presenten a sí mismos o al grupo al que pertenecen de una manera favorable). Si bien todos los métodos de evaluación dietética presentan ventajas y desventajas de acuerdo al fin de investigación, el método de evaluación dietética elegido para este estudio se considera fue el más pertinente debido a que aporta información de la dieta habitual de los individuos. Sin embargo, este método presenta desventajas como la probabilidad de modificación de la ingesta durante 
el tiempo de registro, o la tendencia al sub-registro y a la subestimación de las porciones consumidas, y por ende a la estimación de energía y nutrientes ${ }^{40}$. Intentó limitarse la presencia de este sesgo realizando la pesada de los alimentos ingeridos por parte de los sujetos de estudio que contaban con balanzas de uso doméstico.

Si bien no se considera como una limitación, se debe tener en cuenta el origen de la composición química de los alimentos del software utilizado. Se trató de un software internacional que incluía varias bases de datos de alimentos, entre ellas la de la USDA. No se puede asegurar que las estimaciones representen exactamente lo ingerido, dadas las diferencias en la composición química según la especie $\mathrm{u}$ origen del alimento. A nivel nacional, en Paraguay aún hoy en día no se dispone de una tabla de composición química de alimentos autóctonos. En el presente estudio no se analizó la calidad de la dieta, ni las prácticas alimentarias que influirían sobre la absorción de nutrientes (como forma de preparación de los alimentos, momentos del día en que se consumen anti-nutrientes, entre otros). Tampoco se consideró el porcentaje verdaderamente absorbido de hierro $\mathrm{u}$ otros minerales a partir de los suplementos (varía dependiendo del tipo de hierro del suplemento). Para futuras investigaciones se recomendaría incluir una evaluación acerca de la calidad de la alimentación de los vegetarianos y complementar con análisis bioquímicos de los niveles sanguíneos de algunos nutrientes críticos con el fin de conocer la biodisponibilidad y reserva de los mismos. Otra recomendación consiste en alcanzar un tamaño de muestra suficiente para cada una de las variables alimentarias a evaluarse, y lograr una cantidad homogénea de varones y mujeres por grupos. El trabajo presentó como fortalezas la excelente predisposición de los sujetos de estudio, además de la información muy detallada de la ingesta proporcionada por los mismos.

\section{CONCLUSIONES}

Se presentó una ingesta inferior a los requerimientos en cuanto a energía, carbohidratos, proteínas y grasas en ambos grupos de vegetarianos, además de ingesta muy limitada de calcio y vitamina $B_{12}$ en veganos. Analizando los resultados individualmente y por sexo, no se alcanzó el RDA para hierro en mujeres, y ninguno de los varones ovo-lácteo-vegetarianos cumplió con la IA de fibra. En el caso de minerales como hierro y calcio, fueron las mujeres quienes presentaron ingestas muy por debajo de las recomendaciones. Ninguna vegana cumplió con la $\mathrm{RD}$ de vitamina $\mathrm{B}_{12}$. Al comparar grupos, se hallaron diferencias significativas en el consumo de proteínas y calcio, siendo significativamente menor en los veganos. Lo mismo sucedió con la fibra, siendo significativamente menor en los ovo-lácteo-vegetarianos. La suplementación también fue muy baja. Actualmente, el vegetarianismo en el Paraguay parece ir en aumento, y los profesionales en nutrición tienen un papel insoslayable en el asesoramiento a los individuos que expresen interés en adoptar este tipo de dieta, no sólo en cuanto a los aspectos cuantitativos, sino también en cuanto a prácticas que mejoren la calidad y biodisponibilidad de los nutrientes.

\section{BIBLIOGRAFÍA}

1. Clarys P, Deriemaeker P, Huybrechts I, Hebbelinck M, Mullie $P$. Dietary pattern analysis: a comparison between matched vegetarian and omnivorous subjects. Nutr J. 2013; 12: 1-6.

2. Craig W, Mangels A. Position of the American Dietetic Association: vegetarian diets. I Am Diet Assoc. 2009; 109: 1266-1282.

3. Tuso P, Ismail M, Ha B, Bartolotto C. Nutritional update for physicians: plant-based diets. Perm J. 2013; 17: 61-66.

4. Craig W. Health effects of vegan diets. Am J Clin Nutr. 2009; 89: 1627S-1633S.

5. Chiang JK, Lin YL, Chen CL, Ouyang CM, Wu YT, Chi YC, et al. Reduced Risk for Metabolic Syndrome and Insulin Resistance Associated with Ovo-Lacto-Vegetarian Behavior in Female Buddhists: A Case-Control Study. PLoS One. 2013; 8: e71799.

6. Rizzo N, Sabate J, Jaceldo-Siegl K, Fraser G. Vegetarian dietary patterns are associated with a lower risk of metabolic syndrome: the adventist health study 2. Diabetes Care. 2011; 34: 1225-1227.

7. Suverza-Fernández A, Haua K. The ABCD of the Nutrition Status Assessment. México, McGraw-Hill Interamericana, 2014.

8. Durnin IVGA, Womersley J. Body fat assessed from total body density and its estimation from skinfold thickness: measurements on 481 men and women aged from 16 to 72 years. Br J Nutr. 1974; 32: 77-97.

9. Gallagher D, Heymsfield S, Heo M, Jebb S, Murgatroyd, PR Sakamoto $Y$. Healthy percentage body fat ranges: an approach for developing guidelines based on body mass index. Am J Clin Nutr. 2000; 72: 694-701.

10. Vázquez $M$, Witriw A. Visual food models \& weight/volume ratio tables. UBA editor, Buenos Aires, 1997.

11. Navarro A, Cristaldo P, Andreatta $M$, Muñoz S, Díaz M, Lantieri M. Food Atlas. National University of Córdoba, Córdoba, 2007.

12. World Health Organization. Human energy requirements. Rome, 2001; p. 35-50.

13. Translation of the Guidelines for Data Processing and Analysis of the International Physical Activity Questionnaire (IPAQ) Short and Long Versions. University of Granada, Andalucía: 2005.

14. Carrera Y. International Physical Activity Questionnaire (IPAQ). Rev Enferm Trab. 2017; 7: 49-54.

15. Institute of Medicine (US). Dietary Reference Intakes for Calcium. Washington, D.C., 1997.

16. Institute of Medicine (US). Dietary Reference Intakes for Iron. Washington, D.C., 2001.

17. Institute of Medicine (US). Dietary Reference Intakes for Vitamin B12. Washington, D.C., 1998.

18. Institute of Medicine (US). Dietary reference Intakes for Energy, Carbohydrate, Fiber, Fat, Fatty Acids, Cholesterol, Protein and Amino Acids. Washington, D.C., 2005.

19. Deriemaeker P, Alewaeters K, Hebbelinck M, Lefevre I, Philippaerts $R$, Clarys $P$. Nutritional status of Flemish vegetarians compared with non-vegetarians: a matched samples study. Nutrients. 2010; 2: 770-780.

20. Clarys $P$, Deliens T, Huybrechts I, Deriemaeker P, Vanaelst $B$, De Keyzer W. Comparison of nutritional quality of the 
vegan, vegetarian, semi-vegetarian, pesco-vegetarian and omnivorous diet. Nutrients. 2014; 6: 318-1332.

21. Suárez López M, Kizlansky A, López L. Evaluation of the quality of proteins in food by calculating the amino acid score corrected for digestibility. Nutr Hosp. 2006; 21: 47-51.

22. Rizzo N, Jaceldo-Siegl K, Sabate J, Fraser G. Nutrient profiles of vegetarian and nonvegetarian dietary patterns. J Acad Nutr Diet. 2013; 113: 1610-1619.

23. Blanco A. Macrominerals. In: Micronutrients, vitamins and minerales. Buenos Aires: Promed; 2009; p. 217-280.

24. Killip S, Bennett J, Chambers M. Iron deficiency anemia. Am Fam Physician. 2007; 75: 671-678.

25. Blanco-Rojo $R$, Vaquero MP. Iron bioavailability from food fortification to precision nutrition. A review. Innov Food Sci Emerg Technol. 2019; 51: 126-138.

26. Gaitán D, Olivares M, Arredondo M, Pizarro F. Iron bioavailability in humans. Rev Chil Nutr. 2006; 33: 142-148.

27. Gallego-Narbón A, Zapatera B, Vaquero M. Physiological and dietary determinants of iron status in Spanish vegetarians. Nutrients. 2019; 11: E1734.

28. Rojas D, Figueras F; Durán S. Advantages and disadvantages of being vegan or vegetarian. Rev Chil Nutr. 2017; 44: 218225.

29. Appleby P, Roddam A, Allen N, Key T. Comparative fracture risk in vegetarians and nonvegetarians in EPIC-Oxford. Eur J Clin Nutr. 2007; 61: 1400-1406.

30. Madry E, Lisowska A, Grebowiec P, Walkowiak J. The impact of vegan diet on B-12 status in healthy omnivores: five-year prospective study. Acta Sci Pol Technol Aliment. 2012; 11: 209-212.

31. Smith A, Refsum H. Do we need to reconsider the desirable blood level of vitamin B12? J Intern Med. 2012; 271: 179-182.

32. Nebl J, Schuchardt JP, Ströhle A, Wasserfurth P, Haufe S, Eigendorf J, et al. Micronutrient status of recreational runners with vegetarian or non-vegetarian dietary patterns. Nutrients. 2019; 11: 1-16.

33. Gallego-Narbón A, Zapatera B, Barrios L, Vaquero MP. Vitamin $B 12$ and folate status in Spanish lacto-ovo vegetarians and vegans. I Nutr Sci. 2019; 8: 1-8.

34. Centurión-Bernal EG, González-Acosta AG, Rojas-Pavón MB, Burgos-Larroza RO, Meza-Miranda E. Food knowledge, practices and attitudes of vegetarians in Paraguay. Mem Inst Investig Cienc Salud. 2018; 16: 19-25.

35. Brignardello G, Heredia L, Ocharán M, Durán S. Food knowledge of Chilean vegetarians and vegans. Rev Chil Nutr. 2013; 40: 129-134.

36. Ahmad Fuzi SF, Koller D, Bruggraber S, Pereira DIA, Dainty $J R$, Mushtaq S. A 1-h time interval between a meal containing iron and consumption of tea attenuates the inhibitory effects on iron absorption: A controlled trial in a cohort of healthy UK women using a stable iron isotope. Am J Clin Nutr. 2017; 106: 1413-1421.

37. Thankachan P, Walczyk T, Muthayya S, Kurpad A V., Hurrell RF. Iron absorption in young Indian women: The interaction of iron status with the influence of tea and ascorbic acid. Am J Clin Nutr. 2008; 87: 881-886.

38. Tantamango-Bartley Y, Jaceldo-Sieg/ K, Fan J, Fraser G. Vegetarian diets and the incidence of cancer in a low-risk population. Cancer Epidemiol Biomarkers Prev. 2013; 22: 286-294.

39. Biswas A, Oh Pl, Faulkner GE, Bajaj RR, Silver MA, Mitchell MS et al. Sedentary Time and Its Association With Risk for Disease Incidence, Mortality, and Hospitalization in Adults: A Systematic Review and Meta-Analysis. Ann Intern Med. 2015; 162: 123-132.

40. Ortega RM, Pérez-Rodrigo C, López-Sobaler AM. Evaluation methods of current intake: dietaty record or dietary diary. Rev Esp Nutr Comunitaria. 2015; 21: 34-41. 